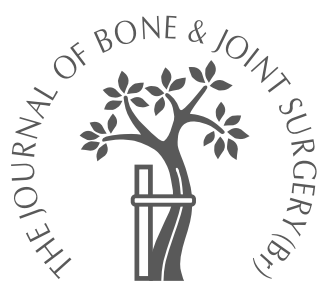

R. A. W. Verhagen, M. Maas, M. G. W. Dijkgraaf, J. L. Tol, R. Krips, C. Niek van Dijk

From the Academic Medical Centre, Amsterdam, The Netherlands

\title{
Prospective study on diagnostic strategies in osteochondral lesions of the talus
}

\author{
IS MRI SUPERIOR TO HELICAL CT?
}

R. A. W. Verhagen, MD, PhD, Orthopaedic Surgeon Department of Orthopaedic and Trauma Surgery, Ziekenhuis Hilversum, PO Box 10016, 1201 DA Hilversum, The Netherlands.

M. Maas, MD, PhD, Radiologist

Department of Radiology

M. G. W. Dijkgraaf, PhD,

Epidemiologist

Department of Clinical

Epidemiology and

Biostatistics

J. L. Tol, MD, PhD,

Orthopaedic Resident

R. Krips, MD, PhD,

Orthopaedic Resident

C. Niek van Dijk, MD, PhD,

Orthopaedic Surgeon

Department of Orthopaedic

Surgery

Academic Medical Centre,

PO Box 22660, 1100 DD

Amsterdam, The

Netherlands.

Correspondence should be sent to Dr R. A. W. Verhagen.

(C)2005 British Editorial

Society of Bone and

Joint Surgery

doi:10.1302/0301-620X.87B1.

$14702 \$ 2.00$

$J$ Bone Joint Surg [Br]

2005;87-B:41-6.

Received 13 June 2003

Accepted after revision

26 May 2004

Our aim in this prospective study was to determine the best diagnostic method for discriminating between patients with and without osteochondral lesions of the talus, with special relevance to the value of MRI compared with the new technique of multidetector helical CT. We compared the diagnostic value of history, physical examination and standard radiography, a $4 \mathrm{~cm}$ heel-rise view, helical CT, MRI, and diagnostic arthroscopy for simultaneous detection or exclusion of osteochondral lesions of the talus. A consecutive series of 103 patients (104 ankles) with chronic ankle pain was included in this study. Of these, 29 with 35 osteochondral lesions were identified. Twenty-seven lesions were located in the talus.

Our findings showed that helical CT, MRI and diagnostic arthroscopy were significantly better than history, physical examination and standard radiography for detecting or excluding an osteochondral lesion. Also, MRI and diagnostic arthroscopy performed better than a mortise view with a $4 \mathrm{~cm}$ heel-rise. We did not find a statistically significant difference between helical CT and MRI. Diagnostic arthroscopy did not perform better than helical CT and MRI for detecting or excluding an osteochondral lesion.

An osteochondral lesion of the talus (OLT) is one of the most important causes of residual pain after an ankle sprain. It is defined as the separation of a fragment of articular cartilage, with or without subchondral bone. ${ }^{1,2}$ Its incidence after an ankle sprain which has been reported as up to $6.5 \%$, is probably underestimated because these lesions often remain undetected. ${ }^{3,4}$

Although initial symptoms may be absent, in chronic cases most patients present with intermittent deep pain in the ankle which increases on weight-bearing. On physical examination signs are often lacking. There may be some minor limitation of movement and synovitis. There is usually no local tenderness.

Particularly at an early stage, an OLT is difficult to visualise by conventional radiography. ${ }^{5,6}$ The frequent absence of radiological changes has led to the use of more sensitive methods of detection. ${ }^{7}$ While some authors prefer additional radiographs such as mortise views with a $4 \mathrm{~cm}$ heel-rise, the lack of precise localisation and extent of the lesion remains a problem. ${ }^{8,9}$ Therefore, others prefer CT, ${ }^{10}$ MRI, ${ }^{11-13}$ or diagnostic arthroscopy. ${ }^{14}$

Currently, the most satisfactory non-invasive diagnostic technique for identifying an OLT is MRI. ${ }^{15,16}$ This is identified as an appropriate investigation for chronic ankle pain by the
American College of Radiology. ${ }^{17}$ However, in our experience, high-resolution helical CT can accurately detect an OLT. Hence, the superiority of MRI over helical CT as the instrument of choice in evaluating an OLT may be questioned. There have been no prospective studies which have compared the efficacy of these two diagnostic methods in the evaluation of osteochondral lesions. ${ }^{18}$ We have, therefore, carried out a prospective study in which the various noninvasive diagnostic methods for detecting an OLT have been compared with diagnostic arthroscopy. Our aim was to determine the diagnostic value of history taking, physical examination (HPX) and standard radiography, the taking of mortise view with a $4 \mathrm{~cm}$ heel-rise (mortise view; 4-cm block), helical CT, MRI, and diagnostic arthroscopy, and to identify the best diagnostic method for detecting an OLT. Our hypothesis was that HPX plus any test would perform better than HPX alone, helical CT and MRI would be better than mortise view, MRI would be better than helical CT and that diagnostic arthroscopy would be better than any other test.

\section{Patients and Methods}

Between October 1997 and May 1999, all patients with chronic ankle pain who presented 
to our outpatient department were consecutively included into a diagnostic protocol, which was approved by the Institutional Review Board. Patients were included if they had failed to respond to conservative treatment for at least six months consisting of analgesics (non-steroidal anti-inflammatory drugs), bracing, and/or physiotherapy. The inclusion and exclusion criteria are given in Table I.

There were 103 patients with a mean age of 33 years (18 to 68$)$. Eighty-two $(79 \%)$ had a history of ankle trauma and $32(31 \%)$ had undergone previous surgery on the ankle. All except one had unilateral involvement. Table II gives the details of the patients. A total of 35 OLTs were identified in 29 patients, of whom $25(86 \%)$ had a history of ankle trauma (23 with an inversion injury and two with a fracture). All patients with an OLT had pain related to weight-bearing felt deep in the ankle. Pre-operative radiological findings showed that 16 ankles had grade 0 osteoarthritic changes, nine grade I and four grade II. ${ }^{19}$

Non-invasive diagnostic procedures. All patients underwent a diagnostic protocol, consisting of routine physical examination, and standard AP and lateral weight-bearing radiographs of both ankles (HPX). After informed consent all patients were scheduled for arthroscopy. In the meantime additional investigations were performed: an AP mortise view (weight-bearing) of both ankles with a $4 \mathrm{~cm}$ heel rise, ${ }^{8}$ CT with multiplanar reconstructions in the sagittal and coronal planes and MRI. High-resolution multidetector helical CT was also performed using a dual-helix CT (Elscint Twin Flash, Haifa, Israel or Picker MX Twin Flash, Cleveland, Ohio). Axial data acquisition was performed with 0.5 $\mathrm{mm}$ slices 3D data set. Both semi-coronal and sagittal reformatted images of $2 \mathrm{~mm}$ were reconstructed.

MRI without intravenous or intra-articular contrast was performed with a 1.5 Tesla vision system (Siemens, Erlangen, Germany), with the ankle placed in a circularly polarised circularly polarised head coil. Although a flexible extremity coil was available the circularly polarised head coil was chosen since it provided the best signal-to-noise ratio. ${ }^{20}$ The imaging protocol consisted of sagittal TSTIR (turbo short tau inversion recovery), T1-weighted SE, sagittal, coronal, and axial FSE (fast spin echo) dual-echo sequences ( $3 \mathrm{~mm}$ ), and DESS (dual echo steady state) 3D with reformatted images in the coronal and axial planes $(2 \mathrm{~mm})$.

All CT and MR scans were separately evaluated by an experienced musculoskeletal radiologist (MM) without knowledge of the patients' history and outcome of the standard radiographs. The radiologist was blinded to the CT results when evaluating MRI, and vice versa.

In contrast to those of plain radiography, the CT and MR scans were not disclosed to the surgeon who performed the arthroscopy. A second orthopaedic surgeon $(\mathrm{CNvD})$ was informed of the outcome of the diagnostic tests and discussed with the patient the type of operative procedure which would be undertaken.

Arthroscopic procedure. The arthroscopic procedures were performed within 12 weeks of inclusion into the study by
Table I. Details of the inclusion and exclusion criteria

Inclusion criteria
Persistent symptoms in the ankle for more than six months
Suspected articular cause (ankle)
Age of patient over 18 years
Exclusion criteria
Suspected extra-articular cause
Systemic disease (rheumatoid arthritis, systemic lupus erythemato-
sus, malignancy, etc)
sus, malignancy, etc)

Table II. Details of the patients

\begin{tabular}{lll}
\hline & Whole group & OLT group \\
\hline Number of patients & 103 & 29 \\
Number of ankles involved & 104 & 29 \\
Number of osteochondral lesions & 35 & 35 \\
$\quad$ Talar & & 21 \\
$\quad$ Talar and tibial & & 6 \\
$\quad$ Tibial & $71: 32$ & 2 \\
Male:female & $51: 53$ & $17: 12$ \\
Right:left ankle & 33.1 (18.3 to 67.7) & $15: 14$ \\
Mean age in years (range) & 82 & 25 \\
History of trauma & 32 & 10 \\
Previous operations & 13 & 6 \\
Arthroscopy & 8 & 3 \\
Ligament reconstruction & 5 & 1 \\
Arthrotomy & 6 & 0 \\
Ankle fracture & & \\
Duration before surgery (mths) & 28 & 6 \\
$\quad 7$ to 12 & 32 & 9 \\
$\quad$ 13 to 24 & 44 & 14 \\
$\quad>24$ &
\end{tabular}

the senior author $(\mathrm{CNvD})$ in an outpatient setting under general or regional anaesthesia with a tourniquet at thigh level using $2.7 \mathrm{~mm}$ and $4.0 \mathrm{~mm} 30^{\circ}$ angle arthroscopes. Standard anteromedial and anterolateral portals were used routinely. The ankles were distracted by a non-invasive distraction system. ${ }^{21}$ After completion of the diagnostic arthroscopy the results and those of the HPX and mortise view were incorporated on a standard form. The results of the other radiological examinations were then disclosed to the surgeon and the arthroscopic examination was continued to confirm and treat a lesion. Treatment included arthroscopic removal of the involved osteochondral segment, curettage and multiple drilling of the base with a Kirschner wire $(1.6 \mathrm{~mm}) .^{22,23}$ The more posteriorly located lesions were treated with the ankle in maximum plantar flexion.

Final diagnosis. Thus, after the diagnostic arthroscopy the results of the additional non-invasive and invasive investigations were evaluated by further arthroscopic examination under the same anaesthesia. The outcome of this further procedure, including the previous information, was regarded as the final diagnosis.

Post-operative care consisted of exercises and progressive weight-bearing for six weeks. The follow-up examinations at one and two years were used to verify the final diagnosis. 
Table III. Data on all patients with osteochondral lesions according to the final diagnosis

\begin{tabular}{|c|c|c|c|c|c|c|c|c|c|c|c|}
\hline \multirow[b]{2}{*}{ Case } & \multirow[b]{2}{*}{ Gender } & \multirow[b]{2}{*}{ HPX* } & \multirow[b]{2}{*}{ MX* } & \multicolumn{2}{|l|}{ CTt } & \multicolumn{2}{|l|}{ MRIt } & \multicolumn{2}{|c|}{ Arthroscopy $\dagger$} & \multicolumn{2}{|c|}{ Final diagnosis } \\
\hline & & & & Talar & Tibial & Talar & Tibial & Talar & Tibial & Talar & Tibial \\
\hline 1 & $M$ & + & + & PM & - & PM & - & PM & - & PM & - \\
\hline 2 & $F$ & - & - & - & - & PM & - & PM & - & PM & - \\
\hline 3 & $F$ & - & - & $A L$ & - & $\mathrm{AL}$ & $A L$ & $\mathrm{AL}$ & - & $\mathrm{AL}$ & $A L$ \\
\hline 4 & $M$ & + & + & - & - & $\mathrm{AL}$ & - & $A L$ & - & $A L$ & - \\
\hline 5 & $M$ & + & + & PM & - & PM & - & PM & - & PM & - \\
\hline 6 & $M$ & + & + & AM & $A$ & AM & AM & AM & AM & AM & AM \\
\hline 7 & $M$ & - & - & - & - & - & - & - & A & - & A \\
\hline 8 & $F$ & - & - & - & $\mathrm{P}$ & - & $P$ & - & - & - & $P$ \\
\hline 9 & $M$ & - & - & AM & - & AM & - & AM & - & AM & - \\
\hline 10 & $M$ & - & + & $\mathrm{AL}$ & - & $\mathrm{AL}$ & - & $\mathrm{AL}$ & - & $\mathrm{AL}$ & - \\
\hline 11 & $M$ & + & + & PL & - & PL & - & PL & - & PL & - \\
\hline 12 & $\mathrm{M}$ & + & + & $\mathrm{AL}$ & $A L$ & $\mathrm{AL}$ & $A L$ & $\mathrm{AL}$ & - & $\mathrm{AL}$ & $A L$ \\
\hline 13 & $M$ & - & + & AM & - & AM & - & AM & - & AM & - \\
\hline 14 & $M$ & + & + & $\mathrm{CL}$ & - & $\mathrm{CL}$ & - & $\mathrm{CL}$ & - & $\mathrm{CL}$ & - \\
\hline 15 & $F$ & + & + & $\mathrm{AL}$ & $A$ & $\mathrm{AL}$ & $A L$ & $\mathrm{AL}$ & - & $\mathrm{AL}$ & $A L$ \\
\hline 16 & $\mathrm{~F}$ & + & + & $\mathrm{CM}$ & - & $\mathrm{CM}$ & - & $\mathrm{CM}$ & - & $\mathrm{CM}$ & - \\
\hline 17 & $F$ & - & - & - & - & PM & - & PM & - & PM & - \\
\hline 18 & $\mathrm{~F}$ & - & - & - & $A$ & - & $A$ & $\mathrm{CL}$ & A & $\mathrm{CL}$ & A \\
\hline 19 & $F$ & - & + & PM & - & PM & - & PM & - & PM & - \\
\hline 20 & $M$ & - & - & - & - & AM & A & AM & A & AM & $A$ \\
\hline 21 & $\mathrm{~F}$ & - & - & PM & - & $=\ddagger$ & $=\ddagger$ & PM & - & PM & - \\
\hline 22 & $\mathrm{~F}$ & + & + & PM & - & PM & - & PM & - & PM & - \\
\hline 23 & $\mathrm{~F}$ & + & + & $\mathrm{AL}$ & - & $\mathrm{AL}$ & - & $\mathrm{AL}$ & - & $\mathrm{AL}$ & - \\
\hline 24 & $M$ & + & + & PM & - & PM & - & PM & - & PM & - \\
\hline 25 & $M$ & + & + & $\mathrm{CL}$ & - & $\mathrm{CL}$ & - & $\mathrm{CL}$ & - & $\mathrm{CL}$ & - \\
\hline 26 & $M$ & - & - & $\mathrm{CM}$ & - & $\mathrm{CM}$ & - & $\mathrm{CM}$ & - & $\mathrm{CM}$ & - \\
\hline 27 & $M$ & + & + & PL & - & PL & - & PL & - & PL & - \\
\hline 28 & $M$ & + & + & $\mathrm{M}$ & - & $\mathrm{M}$ & - & PM & - & PM & - \\
\hline 29 & $F$ & + & + & $\mathrm{CM}$ & - & $\mathrm{CM}$ & - & $\mathrm{CM}$ & - & $\mathrm{CM}$ & - \\
\hline
\end{tabular}

* HPX, history taking physical examination and standard radiography; MX, mortise view; +, positive for an OLT; -, negative for an OLT

† AM, anteromedial; CM, centromedial; PM, posteromedial; $\mathrm{A}$, anterior; $\mathrm{AL}$, anterolateral; $\mathrm{CL}$, centrolateral; $\mathrm{PL}$, posterolateral; $\mathrm{P}$, posterior

$\ddagger=$, not MRI-compatible

Statistical analysis. Based on the final diagnosis the sensitivity, specificity and positive and negative predictive values were calculated for each diagnostic test i.e. HPX, mortise view, helical CT, MRI, and diagnostic arthroscopy. Furthermore, the diagnostic odds ratio (DOR) was calculated. ${ }^{24}$ Although the DOR is not yet a commonly applied statistical measure in radiological or orthopaedic studies, it is very applicable, since it is independent of the underlying prevalence of the diagnosis under investigation. ${ }^{25,26}$ It is calculated as the likelihood ratio of a positive test result divided by the likelihood ratio of a negative test result and measures the discriminatory capacity of a test. ${ }^{25}$ Its value ranges from 0 to infinity. Higher values indicate better test performance and unity indicates no discrimination between diseased and non-diseased persons. ${ }^{26}$

The relative diagnostic odds ratio (RDOR) and its confidence interval (CI) adjusted for test covariance were calculated to compare the discriminatory capacity of different tests in our patients. If the RDOR did not include unity, the tests differed. The width of the CI was determined by the number of multiple comparisons made $(n=4)$ and the common correlations among pairs of tests (all between 0.55 and $0.9) .^{27}$
For analysing statistically significant differences between the various diagnostic methods the RDORs with $98.75 \%$ one-tailed CIs were calculated ( $\alpha=0.0125$, one-tailed).

\section{Results}

Final diagnosis. Table III gives details of all 29 patients with an OLT. Twenty-one had a talar osteochondral lesion, two a tibial osteochondral lesion, and six combined talar and tibial lesions. Seven osteochondral lesions were seen anterolaterally on the talar dome, four anteromedially, two centrolaterally, three centromedially, nine posteromedially and two posterolaterally. Of the tibial osteochondral lesions seven were situated anteriorly and one posteriorly. Methods of diagnosis. The results of the different diagnostic techniques for each patient with an osteochondral lesion are presented in Table III. For the different diagnostic methods the sensitivity, specificity, the positive and negative predictive values, and the DOR are given in Table IV. Intertest comparisons, based on the RDOR and its confidence intervals are listed in Table V.

Using HPX, an OLT was suspected in 23 patients and was correct in 16. In addition, HPX did not lead to suspicion of a lesion in 81 patients, of whom 11 had one (false- 
Table IV. The calculated sensitivity, specificity, positive and negative predictive value, and DOR of the different diagnostic methods for an OLT

\begin{tabular}{|c|c|c|c|c|c|c|}
\hline $\begin{array}{l}\text { Diagnostic } \\
\text { method* }\end{array}$ & Number & Sensitivity & Specificity & $\begin{array}{l}\text { Positive predictive } \\
\text { value }\end{array}$ & $\begin{array}{l}\text { Negative predictive } \\
\text { value }\end{array}$ & DOR \\
\hline HPX & 104 & 0.59 & 0.91 & 0.70 & 0.86 & 14.5 \\
\hline $\mathrm{MX}$ & 104 & 0.70 & 0.94 & 0.79 & 0.90 & 34.2 \\
\hline MDCT & 104 & 0.81 & 0.99 & 0.96 & 0.94 & 334 \\
\hline MRIt & 102 & 0.96 & 0.96 & 0.89 & 0.99 & 608 \\
\hline DA $\ddagger$ & 104 & 1.0 & 0.97 & 0.93 & 1.0 & 709 \\
\hline
\end{tabular}

Table V. The RDOR with the corresponding $98.75 \% \mathrm{Cl}$ for the comparison of the discriminatory capacity of the diagnostic methods for an OLT

\begin{tabular}{lllll}
\hline $\begin{array}{l}\text { Diagnostic } \\
\text { method* }\end{array}$ & Number & RDOR & Lower $\mathbf{C l}$ & Exact p value \\
\hline MX-HPX & 104 & 2.35 & 0.851 & 0.03 \\
MDCT-HPX & 104 & 23.0 & 1.55 & $0.005 \dagger$ \\
MDCT-MX & 104 & 9.78 & 0.653 & 0.03 \\
MRI\#-HPX & 102 & 38.6 & 2.67 & $0.001 \dagger$ \\
MRI-MX & 102 & 15.8 & 1.13 & $0.009 \dagger$ \\
MRI-MDCT & 102 & 1.93 & 0.0665 & 0.3 \\
DA§-HPX & 104 & 56.4 & 3.88 & $0.0004 \dagger$ \\
DA-MX & 104 & 26.2 & 1.89 & $0.003 \dagger$ \\
DA-MDCT & 104 & 4.81 & 0.232 & 0.1 \\
DA-MRI & 102 & 2.81 & 0.187 & 0.2 \\
\hline
\end{tabular}

*MX, mortise view; HPX, history taking physical examination and standard radiography; MDCT, multidetector CT; DA, diagnostic arthroscopy

$\dagger$ statistically significant $(\mathrm{p}<0.0125$, one-tailed $\mathrm{Cl}$ )

$\ddagger \mathrm{N}=102$; two lesions were not MRI-compatible due to screws in the talus and metallic clips in the brain

$\S$ comparisons with DA were corrected for continuity

negative). The DOR equalled 14.5. Compared with HPX (Table V), all other tests performed significantly better, except for the mortise view.

Using the mortise view, an OLT was suspected in 24 patients. This was diagnosed correctly in 19 of 27 talar lesions. Based on the mortise view, five lesions were suspected in 77 patients who did not have one (false-positive) with a DOR of 34.2. In $12(63 \%)$ of these 19 , the lesion was located centrally or posteriorly. Compared with the mortise view (Table V), MRI and diagnostic arthroscopy were statistically better while helical CT was not ( $\mathrm{p}=$ $0.009,0.003$ and 0.03 , respectively).

Helical CT identified 22 OLTs correctly and gave a falsenegative result in five. Two (cases 2 and 4) were classified according to Anderson et al, ${ }^{9}$ for MRI as having a grade I lesion, and two (cases 17 and 20) as having grade-II-Alesions. The other CT false-negative case (case 18) was also false-negative on MRI and classified as stage D (flap present) according to the arthroscopic classification for OLTs. ${ }^{28}$ CT was false-positive in one patient. Previously, this patient had had a fracture of the ankle with local osteoarthritic cysts and interruption of the cartilage. The sensitivity and specificity for helical CT were 0.81 and 0.99 , respectively, with a DOR of 334. Compared with helical
CT (Table V), MRI and diagnostic arthroscopy were not statistically better ( $p=0.33$ and 0.12 , respectively).

One patient was not suitable for MRI because of the presence of screws in the talus and one because of metal clips in the brain. One of these had an OLT. MRI identified 28 OLTs and in three MRI was false-positive. All three had degenerative changes of the talus. Thus, 25 of 26 OLTs were identified correctly. The sensitivity and specificity for MRI were 0.96 and 0.96, respectively, with a DOR of 608 . There was no statistical difference between MRI and diagnostic arthroscopy, with a p value of 0.2 .

Diagnostic arthroscopy identified 29 OLTs of which 27 were correct. Two cases were false-positive. In one of the latter there were degenerative changes of the talus, and in the other local softening of the talar cartilage was present without signs of an OLT on helical CT and/or MRI. Therefore, this lesion was not classified as an OLT. The sensitivity and specificity for diagnostic arthroscopy were 1.0 and 0.97, respectively, with a DOR of 709 .

Eight tibial osteochondral lesions were identified at the final diagnosis. In six a talar lesion was also present, of which five were kissing lesions..$^{29,30}$ No isolated tibial osteochondral lesion was identified on HPX. Helical CT identified five tibial lesions correctly. No false-positive lesions were scored according to the final diagnosis. MRI identified seven of eight tibial lesions. Also no false-positive lesions were scored. On diagnostic arthroscopy four of eight tibial lesions were missed. In three of these (cases 3, 12, and 15) a talar lesion was also present. No false-positive lesions were scored according to the final diagnosis.

The sensitivity and specificity of tibial osteochondral lesions were 0.63 and 1.0 for helical CT, 0.88 and 1.0 for MRI and 0.50 and 1.0 for diagnostic arthroscopy.

Follow-up. At follow-up at two years the results of treatment of the OLTs were excellent or good in 24 patients $(83 \%)$. At follow-up at one and two years there were no changes in the grade of osteoarthritis on the radiographs of all patients.

\section{Discussion}

Plain radiography is useful in the initial evaluation of patients with acute or chronic ankle pain. These initial examinations, however, may not detect or exclude all OLTs. 
In our study routine radiological examination missed $41 \%$ of OLTs. This is comparable with the findings in other studies. ${ }^{4,31}$ Compared with HPX alone, the DOR will more than double by adding a mortise view to the standard radiological examination. Of the non-invasive diagnostic methods, MRI had the highest sensitivity (0.96), but helical CT scanning was more specific $(0.99)$. Both were superior to HPX alone, whereas the mortise view was not.

According to the experimental and clinical experience of Thompson and Loomer ${ }^{8}$ a heel-rise view was developed because a significant number of osteochondral lesions are found posteriorly. ${ }^{32}$ Unexpectedly, only one of the three extra OLTs diagnosed by the mortise view was located posteriorly. In our opinion, this may be explained by the simple fact of an additional radiograph in a different position (plantar flexion) of the talus.

Although MRI is now the most appropriate method to use, ${ }^{15,17}$ our results do not support its presumed superiority in the discriminating patients with or without an OLT. MRI showed a high sensitivity and specificity, but these results were not statistically significantly better than those produced by helical CT with multiplanar reconstructions. This has not hitherto been stated.

Helical CT and MRI have improved the sensitivity and specificity for the detection, exclusion, and characterisation of osteochondral injuries; ${ }^{9}$ MRI identified four chondral lesions, which helical CT did not find. Consequently, MRI provided more certainty than CT that an OLT is indeed absent if MRI is negative. MRI allows visualisation of the extent of the bony involvement and of overlying abnormalities of cartilage, although MRI may not be as useful in showing cortical outlines in comparison with helical CT. ${ }^{33}$

However, the true extent of the OLT may be obscured by concomitant bone-marrow oedema. It is known that the diameter of the lesions measured on MRI can exceed that shown on conventional radiographs. ${ }^{34}$ This is important for the pre-operative planning of drilling, because sufficient perforation of the sclerotic bone is essential.

CT can accurately identify and localise a lesion while defining its extent. ${ }^{35,36}$ In the past CT has been regarded as the reference test by which the final diagnosis was established. ${ }^{37,38}$ The use of helical technology with multiplanar reconstructions has improved diagnostic quality. In our study, $81 \%$ of the OLTs were identified on the CT scan correctly. Only five false-negative cases were found in our study. In four these were simply chondral lesions without subchondral abnormalities. These may be detected when intra-articular contrast was used. Chondral lesions can be missed, since helical CT without intra-articular contrast cannot assess the overlying cartilage. ${ }^{39}$ Our results showed that, given a positive test result, helical CT provided more certainty than MRI that an OLT was actually present. Furthermore, CT had the advantage of a wider availability and a shorter acquisition time compared with MRI.

In 1984 Pritsch, Horoshovski and Farine ${ }^{40}$ first suggested the role of arthroscopy in the treatment of osteo- chondral lesions of the ankle. An advantage of diagnostic arthroscopy over radiological methods is that not only inspection of the cartilage is possible, but by palpation with a probe it is possible to determine if there are soft spots or delaminated areas to pinpoint an OLT. ${ }^{34}$ Furthermore, once the OLT has been detected it can be treated during the same procedure. In our study all OLTs were identified by arthroscopy, but four of eight tibial lesions were missed. In three cases a talar lesion was also found. The tibial lesions were probably overlooked because of the presence of the talar lesions. Since the surgeon was satisfied that the cause of the patients' disorder had been detected no systematic inspection of the tibial plafond was performed.

This phenomenon is known as 'satisfaction of search' ${ }^{41,42}$ It is therefore important to inspect the tibial plafond routinely and systematically even in patients with a talar lesion, for so-called mirror images or kissing lesions. ${ }^{29,30,43}$ We found five kissing lesions in 104 ankles $(5 \%)$, which is comparable with findings in other studies. ${ }^{44}$ Diagnostic arthroscopy was statistically significantly better than HPX and the mortise view. However, it did not show better results than helical CT or MRI in detecting or excluding an OLT.

There are some disadvantages in the design of our study. Ideally, the scoring of the index test and the reference test should be blinded. In our study the reference test consisted of the combination of the initial tests and, as a consequence, had not been scored blindly. We strongly argue that this is reasonable because in practice, information from the combination of tests is the best proxy, of a 'gold standard'. No test available can serve as a 'gold standard' on its own.

We conclude that there is no statistically significant difference in the ability to discriminate patients with or without an OLT between helical CT, MRI, and diagnostic arthroscopy. Helical CT and MRI were statistically significantly better than HPX for detecting or excluding OLTs, while the mortise view was not. Both tests performed very similarly. Helical CT gave more certainty than MRI that an OLT was present, and MRI was more certain in a negative test result.

Contrary to our hypothesis, conventional MRI did not prove to be better than high-resolution multidetector helical CT for the detection or exclusion of osteochondral lesions.

No benefits in any form have been received or will be received from a commercial party related directly or indirectly to the subject of this article.

\section{References}

1. Berndt AL, Harty M. Transchondral fractures (osteochondritis dissecans) of the talus. J Bone Joint Surg [Am] 1959;41-A:988-1020.

2. Alexander AH, Lichtman DM. Surgical treatment of transchondral talar-dome fractures (osteochondritis dissecans): long-term follow-up. J Bone Joint Surg [Am] 1980; 62-A:646-52.

3. Bosien WR, Staples OS, Russell SW. Residual disability following acute ankle sprains. J Bone Joint Surg [Am] 1955;37-A:1237-43.

4. Flick AB, Gould N. Osteochondritis dissecans of the talus (transchondral fractures of the talus): review of the literature and new surgical approach for medial dome lesions. Foot Ankle 1985;5:165-85.

5. Kabbani YM, Mayer DP. Magnetic resonance imaging of osteochondral lesions of the talar dome. J Am Podiatr Med Assoc 1994;84:192-5. 
6. Nelson DW, DiPaola J, Colville M, Schmidgall J. Osteochondritis dissecans of the talus and knee: prospective comparison of MR and arthroscopic classifications. $J$ Comput Assist Tomogr 1990;14:804-8.

7. McCullough RW, Gandsman EJ, Litchman HE, Schatz SL. Dynamic bone scintigraphy in osteochondritis dissecans. Int Orthop 1988;12:317-22.

8. Thompson JP, Loomer RL. Osteochondral lesions of the talus in a sports medicine clinic: a new radiographic technique and surgical approach. Am J Sports Med 1984; 12:460-3.

9. Anderson IF, Crichton KJ, Grattan-Smith T, Cooper RA, Brazier D. Osteochondral fractures of the dome of the talus. J Bone Joint Surg [Am] 1989;71-A:1143-52.

10. Kelberine F, Frank A. Arthroscopic treatment of osteochondral lesions of the talar dome: a retrospective study of 48 cases. Arthroscopy 1999;15:77-84.

11. Bohndorf K. Osteochondritis (osteochondrosis) dissecans: a review and new MRI classification. Eur Radiol 1998;8:103-12.

12. Aerts $\mathbf{P}$, Disler DG. Abnormalities of the foot and ankle: $M R$ imaging findings. AJR Am J Roentgeno/ 1995;165:119-24.

13. DeSmet AA, Fisher DR, Burnstein MI, Graf BK, Lange RH. Value of MR imaging in staging osteochondral lesions of the talus (osteochondritis dissecans): results in 14 patients. AJR Am J Roentgenol 1990;154:555-8.

14. Helgason JW, Chandnani VP. Magnetic resonance imaging arthrography of the ankle. Top Magn Reson Imaging 1998;9:286-94.

15. Sanders RK, Crim JR. Osteochondral injuries. Semin Ultrasound CT MR 2001;22: 352-70.

16. Dunfee WR, Dalinka MK, Kneeland JB. Imaging of athletic injuries to the ankle and foot. Radiol Clin North Am 2002;40:289-312.

17. DeSmet AA, Dalinka MK, Alazraki $\mathbf{N}$ et al. Chronic ankle pain: American College of Radiology: ACR appropriateness criteria. Radiology 2000;215 (Suppl):321-32.

18. Stroud CC, Marks RM. Imaging of osteochondral lesions of the talus. Foot Ankle Clin 2000;5:119-33.

19. van Dijk CN, Verhagen RA, Tol JL. Arthroscopy for problems after ankle fracture. J Bone Joint Surg [Br] 1997;79-B:280-4.

20. Maas M, Dijkstra PF, Akkerman EM. Uniform fat suppression in hands and feet through the use of two-point Dixon chemical shift MR imaging. Radiology 1999; 210:189-93

21. van Dijk CN, Verhagen RA, Tol JL. Technical note: resterilizable non-invasive ankle distraction device. Arthroscopy 2001;17:12.

22. Parisien JS. Arthroscopic treatment of osteochondral lesions of the talus. Am J Sports Med 1986;14:211-17.

23. Verhagen RA, Struijis PA, Bossuyt PM, van Dijk CN. Systematic review of treatment strategies for osteochondral defects of the talar dome. Foot Ankle Clin 2003; 8:233-42.

24. Macaskill P, Walter SD, Irwig L, Franco EL. Assessing the gain in diagnostic performance when combining two diagnostic tests. Stat Med 2002;21:2527-46.

25. Kraemer HC. Evaluation medical tests: objective and quantitative guidelines. Newbury Park, USA: SAGE publications, 1992.
26. Lijmer JG. Evaluation of diagnostic tests: from accuracy to outcome [thesis]. Amsterdam: University of Amsterdam, 2001

27. Siegel S, Castellan NJ. Nonparametric statistics for the behavioural sciences. New York: McGraw-Hill, 1988:320.

28. Cheng MS, Ferkel RD, Applegate GR. Osteochondral lesions of the talus: a radiologic and surgical comparison. In: Ferkel RD, Whipple TL, Burst SE, eds. Arthroscopic surgery: the foot and the ankle. Philadelphia: Lippincott-Raven, Williams \& Wilkins, 1996:152.

29. Lundeen RO. Ankle arthroscopy in the adolescent patient. J Foot Surg 1990;29: 510-15.

30. Canosa J. Mirror image osteochondral defects of the talus and distal tibia. Int Orthop 1994;18:395-6

31. Hepple S, Winson IG, Glew D. Osteochondral lesions of the talus: a revised classification. Foot Ankle Int 1999;20:789-93.

32. Baker CL, Graham JM Jr. Current concepts in ankle arthroscopy. Orthopaedics 1993;16:1027-35.

33. Ferkel RD. Articular surface defects, loose bodies, and osteophytes. In: Ferkel RD, Whipple TL, Burst SE, eds. Arthroscopic surgery: the foot and the ankle. Philadelphia: Lippincott-Raven, Williams \& Wilkins, 1996:145-84.

34. Lahm A, Erggelet C, Steinwachs M, Reichelt A. Arthroscopic management of osteochondral lesions of the talus: results of drilling and usefulness of magnetic resonance imaging before and after treatment. Arthroscopy 2000;16:299-304.

35. Ragozzino A, Rossi G, Esposito S, Giovine S, Tuccillo M. Computerized tomography of osteochondral diseases of the talus dome. Radiol Med (Torino) 1996;92: 682-6 (in Italian).

36. Lundeen RO, Stienstra JJ. Arthroscopic treatment of transchondral lesions of the talar dome. J Am Podiatr Med Assoc 1987;77:456-61.

37. Wells D, Olaff-Solomon J. Radiographic evaluation of transchondral dome fractures of the talus. J Foot Surg 1987;26:186-93.

38. Urman M, Ammann W, Sisler J, et al. The role of bone scintigraphy in the evaluation of talar dome fractures. J Nucl Med 1991;32:2241-4.

39. Vande Berg BC, Lecouvet FE, Malghem J. Frequency and topography of lesions of the femorotibial cartilage at spiral CT arthrography of the knee: a study in patients with normal knee radiographs and without history of trauma. Skeletal Radiol 2002; 31:643-9.

40. Pritsch M, Horoshovski H, Farine I. Ankle arthroscopy. Clin Orthop 1984;184:137-40.

41. Samuel S, Kundel HL, Nodine CF, Toto LC. Mechanism of satisfaction of search: eye position recordings in the reading of chest radiographs. Radiology 1995;194: 895-902.

42. Ashman CJ, Yu JS, Wolfman D. Satisfaction of search in osteoradiology. AJR Am J Roentgenol 2000;175:541-4.

43. Sijbrandij ES, van Gils AP, Louwerens JW, de Lange EE. Posttraumatic subchondral bone contusions and fractures of the talotibial joint: occurrence of "kissing" lesions. AJR Am J Roentgenol 2000;175:1707-10.

44. Labovitz JM, Schweitzer ME. Occult osseous injuries after ankle sprains: incidence, location, pattern, and age. Foot Ankle Int 1998;19:661-7. 